\title{
26 Welche Hilfestellung benötigen Behinderte in Schadensfällen? Target Group Intervention Program-Disability
}

\author{
Robert Bering, Florentine Gerwinn, Claudia Schedlich und Gisela Zurek
}

Mit der Verabschiedung der UN-Behindertenrechtskonvention haben wir uns der Gleichstellung von behinderten Menschen neu verpflichtet. Bisher findet dieser Aspekt in den gängigen Multidisciplinary Guidelines (MG) zur Psychosozialen Notfallversorgung (PSNV) nur wenig Berücksichtigung. Wir stellen uns die Frage, welche Empfehlungen im Umgang mit behinderten Menschen in der Psychosozialen Akuthilfe, der Notfallmedizin und der mittel- und langfristigen psychosozialen Versorgung auszusprechen sind. Hierbei haben wir folgenden methodischen Ansatz: Auf der Grundlage des Target Group Intervention Program (TGIP), das im Rahmen von Förderungen der Europäischen Kommission entwickelt worden ist, weisen wir auf Besonderheiten hin, die im Umgang mit behinderten Menschen zu berücksichtigen sind. Hierbei differenzieren wir die Akutintervention vom mittel- und langfristigen Prozess und differenzieren die sogenannte Selbstheiler-, Wechsler und Risikogruppe. In der Notfallmedizin steht sicherlich die Akutversorgung im Mittelpunkt. Unser Beitrag ist nicht nur an Notärzte und Rettungssanitäter gerichtet, sondern schließt auch Notfallseelsorger und Notfallpsychologen sowie professionelle Helfer der mittelund langfristigen psychosozialen Versorgung ein.

\subsection{Einführung}

Wir können davon ausgehen, dass die Mehrzahl der Betroffenen auf eine Schadenslage, die mit notfallmedizinischen Einsätzen in Verbindung stehen, mit einer vorübergehenden Stressreaktion reagieren (vgl. NATO \& OTAN 2008: 39). Hierzu zählen wir z.B. schwere Verkehrsunfälle, Naturkatastrophen, Terroranschläge und andere Großschadenslagen. Aus klinischer Sicht entwickelt sich oft eine akute Belastungsreaktion (F43.o), die sich bei dem weit überwiegenden Teil der Betroffenen spontan rückläufig entwickelt. Die Betroffenen benötigen in erster Linie praktische Hilfe, 
Wiedererstellung ihrer sozialen Bezugssysteme und bedarfsorientierte psychosoziale Akuthilfen zur Rückkehr in die Normalität. Ein Teil der Betroffenen entwickelt längerfristige psychische Beeinträchtigungen. Der Anteil wird auf $25 \%$ geschätzt (vgl. NATO \& OTAN 2008: 36), wobei in Abhängigkeit von der Schadensereigniskonstellation mit einer großen Schwankungsbreite zu rechnen ist.

Im ICD-10 werden anhaltende Reaktionen auf schwere Belastungen unter Anpassungsstörung (F43.2) und Posttraumatischer Belastungsstörung (PTBS, F43.1) klassifiziert. Da sich auch Depressionen, Angststörungen, somatoforme Störungen oder Abhängigkeitserkrankungen manifestieren können, sprechen wir von Verlaufstypen einer Belastungsstörung. Die Internationale Klassifikation der Funktionsfähigkeit, Behinderung und Gesundheit (ICF, 2005) ermöglicht die Berücksichtigung von Kontextfaktoren, die sich durch Großschadenslagen schlagartig verändern können. Es können mentale Funktionsstörungen und Beeinträchtigungen der Aktivität und Teilhabe als Folge auftreten.

Mit der Verabschiedung der UN-Behindertenrechtskonvention (UN-BRK, 2009) haben wir uns der Gleichstellung von behinderten Menschen neu verpflichtet. Bisher findet dieser Aspekt in den gängigen Multidisciplinary Guidelines (MC) zur Psychosozialen Notfallversorgung (PSNV) nur wenig Berücksichtigung. Es stellt sich die Frage, welche Empfehlungen wir für diese Gruppe aussprechen können? Wir stützen uns auf das Target Group Intervention Program (TGIP), das wir als Kriseninterventionsprogramm aus den Schlussfolgerungen der sogenannten „Debriefingkontroverse“ entwickelt haben (vgl. Bering 2011). Das TGIP folgt dem Ansatz, alle Interventionen aus der Perspektive von Betroffenen abzuleiten, die ein hohes Risiko haben, eine Psychotraumafolgestörung zu entwickeln. Die Identifikation einer Risikogruppe erfordert die Kenntnis von spezifischen Risikofaktoren und die Entwicklung von Instrumenten, die in der Lage sind, diese zu erfassen. Hierzu haben wir den sogenannten Kölner Risiko Index (KRI) in verschiedenen Versionen entwickelt (Bering u. Fischer 2005). Vor diesem Hintergrund basiert unser Konzept auf dem

- Prozessverlauf von Belastungsstörungen

- Risikogruppenidentifizierung

- Anpassung des Konzepts auf verschiedene Situationstypologien.

Allerdings stellen wir fest, dass bisher kaum darüber nachgedacht wurde, wie die besondere Bedarfslage von Behinderten zu berücksichtigen ist. Auch die internationalen MG zur Early Intervention haben dieses Problem kaum bedacht. Aus diesem Grund stellen wir im ersten Schritt die Grundzüge des TGIP dar, um in einem zweiten Schritt die besondere Bedarfslage von behinderten Menschen zu reflektieren.

Ersthelfer, Polizisten, Rettungsassistenten, Ärzte und Feuerwehrmänner müssen sich in verschiedenen Schadenslagen unter besonders schwierigen Bedingungen auf behinderte Menschen einstellen. Aus diesem Grund müssen wir die oben charakterisierte Herangehensweise um die Berücksichtigung der spezifischen Bedarfslage von Menschen mit Behinderungen erweitern.

\subsection{Target Group Intervention Program}

Die Interventionsplanung orientiert sich am Prozessverlauf (Zeitkriterium) und am Risikoprofil der Betroffenen. Mit anderen Worten: Es stellt sich die Frage, zu welchem 


\section{Ablaufplan der zielgruppenorientierten Intervention}

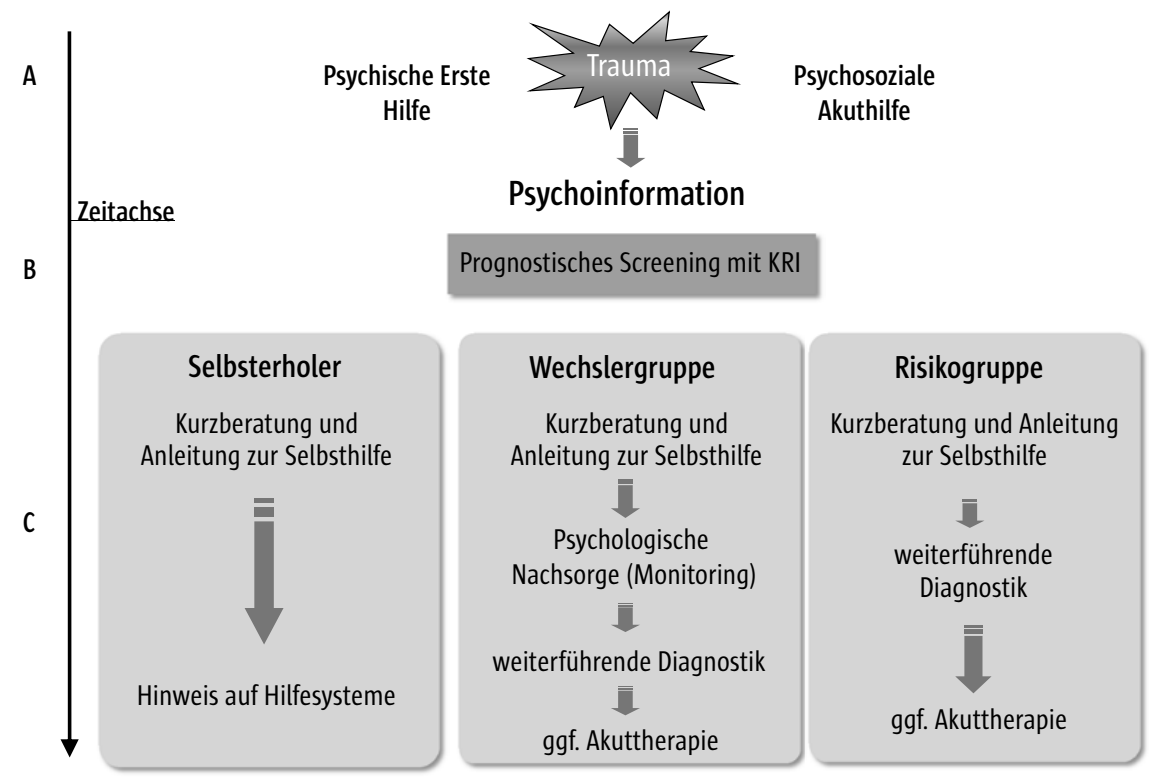

Abb. 16 Target Group Intervention Program. Die Art der Intervention ist vom Prozessverlauf der Belastungsstörung abhängig. In der Phase A steht die Akutversorgung im Mittelpunkt. Die Phase B beschreibt den Übergang von der Schock-zur Einwirkungsphase. Im Mittelpunkt der Phase B stehen die Psychoinformation und das Screening mit dem Kölner Risikoindex. Die Phase C leitet in eine abgestufte Interventionsplanung für die Selbsterholer-, Wechsler- und Risikogruppe ein.

Zeitpunkt welche Intervention erfolgen sollte? Um diese Frage zu beantworten, werfen wir einen Blick auf den Zeitstrahl in Abbildung 16, der in die Akut- (A), die Übergangs- (B) und die Einwirkungsphase (C) unterteilt ist.

Darüber hinaus stellt sich die Frage, welche Interventionen für die unterschiedlichen Kontrastgruppen geeignet sind. Hierzu werden die aufgeführten Maßnahmen aus dem Standardablaufplan (s. Abb. 16) in risikoabhängige und risikounabhängige Module des TGIP unterteilt (s. Tab. 28). Das Ziel ist es, die Ablaufpläne so zu flexibilisieren, dass sie möglichst jede Konstellation berücksichtigen können.

Unabhängig von Einsatzbedingungen, Interventionszeitpunkt und individuellem Risikoprofil gelten basale Maßnahmen, die im Rahmen der sekundären Prävention durchgeführt werden. Hierzu zählen die psychosoziale Akutversorgung Betroffener durch primäre Sicherung, psychologische Erste Hilfe und psychosoziale Akuthilfe (Phase A). Erst wenn die Schockphase abgeklungen ist, erfolgt eine Psychoinformation, die u.a. das prognostische Screening mit dem KRI vorbereitet. Die Ergebnisbesprechung und Kurzberatung schließt die Übergangsphase (B) ab.

Zu den risikoabhängigen Interventionsmodulen gehören das Monitoring, die weiterführende Diagnostik, die Einzelberatung, die Angehörigenberatung und die TraumaAkuttherapie. Auf dem Zeitstrahl sind diese Interventionen der Phase C zuzuordnen. 
26 Welche Hilfestellung benötigen Behinderte in Schadensfällen?

Target Group Intervention Program-Disability

Tab. 28 Module der zielgruppenorientierten Intervention (modifiziert nach Schedlich et al. 2008)

\begin{tabular}{ll} 
Risikounabhängige Maßnahmen & Risikoabhängige Maßnahmen \\
\hline psychische Erste Hilfe & Monitoring \\
psychosoziale Akuthilfe & weiterführende Diagnostik \\
administrative und rechtliche Hilfen & traumazentrierte Fachberatung \\
Unterstützung bei der Organisation von Ritualen/ & Angehörigenberatung \\
Gedenkfeiern & Trauma-Akuttherapie \\
Psychoinformation & \\
prognostisches Screening mit dem Kölner & \\
Risikoindex & \\
Ergebnisbesprechung und Kurzberatung im & \\
Einzelsetting & \\
Anleitung zur Selbsthilfe & \\
\hline Links sind Maßnahmen aufgeführt, die unabhängig vom individuellen Risikoprofil durchgeführt werden \\
sollten. Rechts sind Maßnahmen und Interventionen aufgeführt, die in Abhängigkeit vom erstellten \\
Risikoprofil durchgeführt werden.
\end{tabular}

Somit orientiert sich die Platzierung der Interventionsmodule am Zeitstrahl und am Risikoprofil der Betroffenen. Bisher haben wir uns noch nicht geäußert, was genau den Betroffenen hilft. Hierzu verweisen wir auf die fünf Prinzipien von Hobfoll et al. (2007), die uns Orientierung geben, mit welcher Haltung alle Interventionsmodule durchzuführen sind. Sie lauten:

- Fördere das Sicherheitsgefühl,

- Fördere Beruhigung,

- Fördere das Gefühl von eigener und kollektiver Handlungsfähigkeit

- Fördere Kontakt und Anbindung und

- Fördere das Gefühl von Hoffnung

Diese Prinzipien gelten als Richtlinie für alle Berufsgruppen, die notfallmedizinisch und in die psychosoziale Versorgung eingebunden sind. In unserem Modell gehen wir davon aus, dass diese Prinzipien auch im Umgang mit Behinderten richtungsweisend sind. Um zu einer weiteren Differenzierung zu gelangen, ist es hilfreich, zwischen Maßnahmen zu unterscheiden, die unabhängig und abhängig vom individuellen Risikoprofil empfohlen werden. Berufsgruppen der Notfallmedizin sind in unserem Modell überwiegend der Akutphase zuzuordnen. Ist diese Phase bewältigt, so erfolgt die weitere Betreuung in der Regel durch psychosoziale Akuthelfer (Notfallseelsorger, Kriseninterventionsteams oder Notfallpsychologen). Wir definieren diese Berufsgruppen also explizit als Bestandteil einer notfallmedizinischen Konzeption.

\subsubsection{Risikounabhängige Interventionen}

\section{Psychosoziale Akuthilfe (Phase A)}

In der Akutsituation gelten zunächst für alle Betroffenen - unabhängig von ihrem Risikoprofil - Maßnahmen der primären Versorgung. Hierzu gehören:

- Sicherung und Schutz für die Betroffenen

- medizinische Versorgung 
- Distanzierung vom Ort des Ereignisses

- psychische Erste Hilfe

- psychosoziale Akuthilfe

Die Vermittlung von Informationen beschränkt sich zu diesem Zeitpunkt auf die Transparenz des Vorgehens (medizinische Maßnahmen, Aufenthaltsmöglichkeiten, Ansprechpersonen etc.), beruhigendes Einwirken und erste Informationen zu Möglichkeiten der Selbstberuhigung. Fragen der betroffenen Personen über die Ursachen der Schadenslage werden, soweit bekannt, beantwortet. Informationen müssen klar, kurz und verständlich vermittelt werden, da in der Schocksituation die Aufnahmekapazität eingeschränkt, die Wahrnehmung verzerrt (z.B. Amnesien, Tunnelblick, Zeitlupenerleben) und Handlungsautomatismen gegeben sind (z.B. „Totstellreflex“). Diese Phänomene werden unter dem Fachbegriff der peritraumatischen Dissoziation zusammengefasst.

\section{Psychoinformation (Phase B)}

Die Psychoinformation ist die sachliche Vermittlung von Informationen zu psychischen Phänomenen und Prozessen in unterschiedlichen Kontexten. Zeitlich ist die Psychoinformation in die Phase B, d.h. 2 bis 3 Tage nach dem psychotraumatischen Ereignis, eingeordnet. Bei der Psychoinformation über seelische Verletzungen werden psychotraumatische Verlaufsformen, Möglichkeiten zur Ressourcenstärkung und Hilfsangebote vorgestellt. Ziel einer jeden Psychoinformation ist die Stabilisierung durch Wissenszuwachs, die Erhöhung der Compliance und die individuelle Befähigung zur adäquaten Einschätzung von Risikoverläufen. Die Psychoinformation bei Psychotraumen unterstützt die kognitive Erfassung und Einordnung emotionalen Erlebens und unerklärlicher Verhaltensweisen. Sie wirkt somit dem überwältigenden Gefühl von unkontrollierbaren Sinneseindrücken und Handlungsimpulsen entgegen und erleichtert die Distanzierung von überflutenden Gefühlen. Je nach Interventionszeitpunkt und Setting (Einzel- oder Gruppensetting) variieren die inhaltlichen Schwerpunkte.

In der Regel besteht, gerade bei größeren Schadensfällen, ein ausgeprägtes Informationsbedürfnis bei direkt und indirekt Betroffenen.

Informationen werden gewünscht über Ereignishergang, -umfang, Auslösesituationen, Fragen der Verantwortlichkeit, Anzahl der verletzten und verstorbenen Personen etc. Dies erfordert die Anwesenheit zumindest einer Person, die umfassend über Ereignishergang und -folgen informiert ist. Die Informationsvermittlung unterstützt bei den Betroffenen die kognitive Integration z.T. fragmentierter Erinnerungen in eine Gesamtsituation. Um Belastungseffekte in der Cruppe zu minimieren, sollte Folgendes beachtet werden: Informationen werden sachlich vermittelt, in einer Art Vortragsform, was die emotionale Distanzierung unterstützt. Die Informationen müssen knapp, klar und verständlich formuliert sein. Zu komplexe Inhalte überfordern, können nicht längerfristig gespeichert werden, und es besteht die Gefahr, dass die Betroffenen ,abdriften'. Traumaexplorative Elemente und der Austausch traumabezogener Emotionen sind in der Gruppe unbedingt zu vermeiden, da dies insbesondere für Risikopersonen einen retraumatisierenden Effekt haben kann. Werden traumaexplorative Elemente 
mit ausgeprägter Emotionalität von Teilnehmern in die Gruppe eingebracht, werden diese mit Hinweis auf die Überforderung in der Cruppe behutsam gestoppt und auf den Einzelkontakt verwiesen. Ein Austausch in der Cruppe wird vornehmlich gefördert in Bezug auf ressourcenfördernde Maßnahmen. Hier können die Teilnehmer angeregt werden, erfolgreiche individuelle Strategien der Selbststabilisierung mitzuteilen. Ohne die Perspektive weiterführender Maßnahmen kann eine überwiegend sachliche Informationsvermittlung und das Begrenzen eines emotionalen Austausches als ,im Stich gelassen werden' erlebt werden. Zur manualisierten Darstellung der Durchführung einer Psychoinformation verweisen wir auf Zurek et al. (2008).

\section{Prognostisches Screening von Risiko- und Schutzfaktoren (Phase B/C)}

Im Anschluss an die Psychoinformation wird in der Übergangsphase (Phase B) das prognostische Screening mit dem Kölner Risiko Index (KRI) zur differenzierten Einschätzung des individuellen Risikoprofils durchgeführt. In der Literatur werden mit dem Begriff des „Screenings“ unterschiedliche Konzepte verbunden (IMPACT 2007). Wir verstehen darunter die individuelle Sichtung, in welchem Gleichgewicht Risikound Schutzfaktoren zueinander stehen.

Die Voraussetzung für eine zielgruppenorientierte Vorgehensweise ist die Kenntnis und Identifikation von Risikofaktoren von Psychotraumafolgestörungen. Aus unseren Validierungs- und Intervalidierungsstudien haben sich generelle Risikofaktoren ableiten lassen. Es handelt sich um die Faktorengruppen: Lebensgeschichte, objektive Situationsfaktoren, subjektive Situationsfaktoren, Risiko- und Schutzfaktoren, die im Verlaufsmodell der Psychotraumatisierung dargestellt sind.

$\mathrm{Zu}$ den lebensgeschichtlichen Faktoren gehören z.B. die prätraumatische sozio-emotionale Situation des Betroffenen und vorausgegangene traumatische Erfahrungen. Emotionale Probleme, Schwierigkeiten und Belastungen in Beziehungen, innerhalb der Familie oder auch berufliche Unzufriedenheit oder Perspektivlosigkeit können das Erkrankungsrisiko verstärken. Zum anderen erhöhen vorhergehende traumatisierende Erfahrungen im Lebenslauf das Risiko einer traumatischen Symptombildung. Besonders drastisch können sich die Folgen entwickeln, wenn eine vorherige traumatische Erfahrung nicht verarbeitet, sondern im traumatischen Prozess nur traumakompensatorisch kontrolliert wurde. Durch die erneute Traumatisierung können vorherige Traumaschemata reaktiviert und verstärkt werden.

Zu den ereignisbezogenen Risikofaktoren zählen die objektiven und subjektiven Situationsfaktoren. Zu den objektiven Situationsfaktoren werden die Art des belastenden Ereignisses, die Schwere und Dauer der traumatischen Einwirkung, körperliche Verletzungen und die Bekanntheit des Täters gezählt. Zu den subjektiven Situationsfaktoren gehören die erlebte Lebensbedrohlichkeit und das Ausmaß der peritraumatischen Dissoziation. Je stärker während der situativen Einwirkung dissoziiert wurde, umso schwerer ist die traumatische Erfahrung rekonstruierbar und der bewussten Verarbeitung zugänglich.

Zu den Risikofaktoren im Traumaverlauf gehören vor allem negative Erfahrungen mit der privaten Umwelt, am Arbeitsplatz und mit öffentlichen Funktionsträgern. Eine verständnislose soziale Umwelt, mangelnde Anerkennung der Schwere der Belastung, die Aufforderung zu ,vergessen“ oder ,sich zusammenzureißen“ und das Drängen auf schnelle Wiederherstellung des prätraumatischen Funktionsniveaus können die traumatische Einwirkung verstärken und verlängern. So gilt im Gegenzug als einer der 
wesentlichen Schutzfaktoren, der die Verarbeitung in der Erholungsphase günstig beeinflusst, ein verständnisvolles und gewährendes soziales Umfeld. Ein weiterer Belastungsfaktor für Betroffene liegt häufig in dem Kampf um institutionelle Anerkennung der traumatischen Einwirkung und der daraus resultierenden Folgen, so z.B. bei der Frage der Kostenübernahme für Behandlungen. Die Erholungsphase sollte in jedem Fall durch Maßnahmen wie Ruhe, Entspannung und Fernhalten von der traumatisierenden Umgebung unterstützt sein. Generell sollten Stress und zusätzliche Belastungen vermieden werden.

Die Validierung des KRI stellt die Beziehung her zwischen den Faktorengruppen und der Entwicklung bzw. Aufrechterhaltung von Symptomen im Prozessverlauf einer Belastungsstörung.

In der Instruktion zum KRI sind die Betroffenen darauf hinzuweisen, dass das Ergebnis des Fragebogens maßgeblich ist für die Planung weiterer notwendiger Unterstützungsmaßnahmen. Gerade in Gruppen, bei denen eine ausgeprägte Tendenz zur Dissimulation zu vermuten ist, sind Formulierungen zu vermeiden, die die Erstellung des individuellen Risikoprofils betonen. Es empfiehlt sich zu sagen: „Die Beantwortung des Fragebogens unterstützt uns einzuschätzen, welche Angebote gemacht werden können, um die belastende Erfahrung möglichst schnell zu verarbeiten“. Die Information über die vertrauliche Behandlung der Fragebogenergebnisse und der Hinweis auf die Schweigepflicht sind unbedingt erforderlich, um eine Mitarbeit der Betroffenen zu gewährleisten. Der KRI kann bei geringer Personenanzahl im Einzelkontakt als Interview durchgeführt werden, was eine unmittelbare Einschätzung und Rückmeldung des individuellen Risikos und die Besprechung geeigneter Maßnahmen erlaubt. Bei größeren Gruppen von Betroffenen oder geringer Personalkapazität kann der KRI auch als Selbstbeurteilungsfragebogen in Gruppen ausgefüllt werden. Das individuelle Risikoprofil soll jedem Betroffenen jedoch immer im Einzelkontakt mitgeteilt werden. Nur so kann einer Stigmatisierung in der Gruppe und Dissimulationseffekten vorgebeugt werden.

Abhängig vom erreichten Punktwert im KRI werden die Betroffenen einer der Gruppen zugeordnet: der Selbsterholer-, der Wechsler- oder der Risikogruppe. Bei den Selbsterholern ist mit großer Wahrscheinlichkeit davon auszugehen, dass sie das Ereignis im Rahmen ihres natürlichen Verarbeitungsprozesses mit eigenen Ressourcen und Potenzialen bewältigen. Die Gruppe der Wechsler steht sozusagen ,auf der Kippe‘. Entscheidend dafür, ob der traumatische Verlauf in die Erholungsphase oder in eine Chronifizierung übergeht, ist das Ausmaß zusätzlicher sozialer und institutioneller Belastungen nach dem Ereignis. Die Personen der Risikogruppe weisen ein hohes Risiko auf, eine chronifizierende PTBS oder andere psychiatrische oder psychosomatische Beschwerdebilder zu entwickeln. Bei der Anwendung des KRI sollten wir Folgendes bedenken: In der akuten Schockphase ist die Anwendung obsolet; in der späten Einwirkungsphase ideal. Auch wenn die Anwendung nicht immer möglich ist, so beeinflusst das Wissen um Risikofaktoren den Umgang mit den Betroffenen erheblich. Für die genauere Beschreibung zur Durchführung des Kölner Risikoindex verweisen wir auf das Manual von Bering et al., 2008, das frei zugänglich ist.

\section{Ergebnisbesprechung und Kurzberatung im Einzelsetting (Phase B/C)}

Nach dem prognostischen Screening mit dem KRI wird im Einzelkontakt mit jedem Betroffenen sein persönliches Ressourcen- und Risikoprofil besprochen. Diese Kurz- 
beratung und Prognostik sollen erst nach Abschluss der Schockphase (zwei bis drei Tage nach dem Ereignis) erfolgen. Der persönliche Kontakt erlaubt dem Berater auch die kurze Exploration potenzieller Risiko- und Schutzfaktoren, die Erfassung individueller Ressourcen für die postexpositorische Zeit und einen ersten Eindruck individueller traumakompensatorischer Strategien. Je nach verfügbarem Zeitrahmen haben die Betroffenen im Einzelsetting die Möglichkeit, das Erleben der traumatisierenden Erfahrung anzusprechen. Der Berater sollte aber eine emotionale Vertiefung in diesem Kontext verhindern, ressourcenorientiert intervenieren und auf weitere Kontaktmöglichkeiten verweisen.

Wesentlich ist in der Kurzberatung die Besprechung notwendiger und möglicher Unterstützungsmaßnahmen, die ausführlich erklärt werden sollen. Die Transparenz bezüglich notwendiger Maßnahmen kann langfristig eine höhere Compliance bewirken. Zum Abschluss des Einzelkontaktes können ,Merkblätter' mit Kontaktadressen ausgehändigt werden. Insbesondere bei Schadensereignissen, die auf eine mutmaßliche Straftat zurück zu führen sind, ist die Erstberatung eine wichtige Weichenstellung, um den Zugang zu Opferschutzambulanzen zu vermitteln.

\section{Anleitung zur Selbsthilfe (Phase B/C)}

Eine weitere Maßnahme, die allen Kontrastgruppen angeboten wird, ist die Anleitung zur Selbsthilfe. Den Betroffenen werden Techniken zur Selbstberuhigung und Distanzierung von überflutendem traumatischem Material vermittelt. Hierzu gehören Distanzierungsübungen (z.B. Ablenken durch Rechnen, Sehen-Hören-Empfinden), imaginative Techniken (z.B. Lichtstromtechnik, sicherer innerer Ort, innere Helfer), Entspannungsübungen und Hinweise zu stressreduzierenden Verhaltensweisen (Fischer 2003; Reddemann 2001). Diese Techniken unterstützen den natürlichen Verarbeitungsprozess und fördern den Übergang in die Erholungsphase. Durch die Übungen vermögen die Betroffenen die Erfahrung zu machen, dass sie ihre Emotionen und Gedanken beeinflussen können.

\section{Die Möglichkeit der kognitiven und emotionalen Kontrolle wirkt dem traumati- schen Erleben von Kontrollverlust und Hilflosigkeit entgegen.}

Erfahrungsgemäß vermögen Betroffene die angebotenen Techniken unterschiedlich gut zu nutzen. Damit jeder die persönlich am besten verwertbaren und passenden Techniken auswählen kann, ist es sinnvoll, ein möglichst breites Spektrum von Techniken anzubieten. Da Zeitgründe dem oft eine Grenze setzen, ist hier noch einmal der Hinweis auf die Selbsthilfebroschüren und Audioversionen mit Übungsanleitun-

gen besonders wichtig. Dort finden die Betroffenen einen umfassenden Katalog von Übungen, die sie ausprobieren und ihren individuellen Vorlieben entsprechend auswählen können.

\subsubsection{Risikoabhängige, zielgruppenbezogene Interventionen (Phase C)}

Nach der Akutversorgung, der Psychoinformation, der Prognostik, der Kurzberatung und der Anleitung zur Selbsthilfe unterscheiden sich die angebotenen Unterstüt- 
zungsmaßnahmen in Abhängigkeit vom jeweiligen Risikoprofil, die sich in der Regel bis zu 6 Wochen nach dem Ereignis erstrecken. Ausnahmen bilden Psychotherapien im engeren Sinne des mittel- und langfristigen Prozesses.

\section{Zielgruppenorientierte Intervention bei den Selbsterholern}

Für die Cruppe der Selbsterholer können die bis zu diesem Zeitpunkt angebotenen Maßnahmen zur Unterstützung des Verarbeitungsprozesses - erste Stabilisierung, Psychoinformation, Kurzberatung und Anleitung zur Selbsthilfe - ausreichen. Hilfreich sind in jedem Fall die Sensibilisierung der Betroffenen für chronifizierende Verlaufsformen und der Hinweis auf Unterstützungsangebote. Ein weiterer Kontakt nach zwei bis drei Wochen - persönlich oder telefonisch - kann angeboten werden. Eventuell ungünstige Verlaufsformen und zusätzliche Belastungen können dann erfragt und bei Bedarf weitere Unterstützungsmaßnahmen angeboten werden. Der Umgang mit einem Selbsterholer zielt darauf ab, dem Betroffenen die Sicherheit zu vermitteln, dass bei unerwarteten Komplikationen Helfer zur Verfügung stehen.

\section{Zielgruppenorientierte Intervention bei der Wechslergruppe}

Betroffene, die in die Cruppe der Wechsler eingestuft werden, benötigen eine Nachsorge im Sinne eines begleitenden Monitorings. Hierbei liegt der Fokus auf der Gefahr der Risikopotenzierung durch belastende Lebensumstände. Weiterführende Interventionen - wie Beratung oder Traumaakuttherapie - können bei progredienter Entwicklung frühzeitig angeboten werden. Die regelmäßige Kontaktaufnahme in den Wochen und Monaten nach dem Ereignis soll, sei dies telefonisch oder persönlich, proaktiv durch Fachkräfte der psychosozialen Hilfen erfolgen. Traumaimmanente Vermeidungstendenzen mit einer depressiven Entwicklung, Verleugnung und Bagatellisierung können hierdurch erkannt und durch gezielte Interventionen gelöst werden.

\section{Hilfreich sind konkrete Termine für Telefonate einer persönlichen Kontaktaufnah- me, die auf den Prozessverlauf abgestimmt ist und den biphasischen Verlauf von Vermeidung und Intrusionszyklus berücksichtigt. Dies vermittelt Sicherheit und kann einen psychischen ,Anker' für die Betroffenen bedeuten.}

Werden Termine nicht wahrgenommen, obliegt es der Bezugsperson, aktiv Kontakt aufzunehmen und zu klären, aus welchem Grund Termine nicht wahrgenommen wurden. Nur so können eventuell Verleugnungs- und Rückzugstendenzen rechtzeitig erkannt und abgefangen werden.

\section{Zielgruppenorientierte Intervention bei der Risikogruppe}

Bei den Risikopersonen sollte in jedem Fall eine umfassende individuelle Beratung in mehreren Beratungskontakten erfolgen. Eine differenzierte klinische Diagnostik kann klären, ob sich eine PTBS oder andere komorbide Störungen entwickelt haben, die ausschließlich von Psychologischen Psychotherapeuten oder Fachärzten durchgeführt werden sollte. Sollte das Risiko zeigen, dass sich eine psychische Störung entwickelt, so ist eine Traumaakuttherapie zu empfehlen. Sinnvoll ist es, wenn mög- 
lich, auch die Angehörigen in die Beratung und Behandlung zu integrieren, um so einen der wesentlichen protektiven Faktoren - die soziale Unterstützung - zu stärken.

\subsection{Welche Hilfestellung benötigen Behinderte in Schadensfällen?}

Zunächst müssen wir uns vor Augen führen, dass der Umgang mit behinderten Menschen in der PSNV noch nicht systematisch konzeptionalisiert ist. Wir möchten erste Schritte gehen, indem wir das TGIP unter dem Aspekt der besonderen Bedürfnislage behinderter Menschen reflektieren. Wir müssen also den Prozessverlauf von Belastungsstörungen und die Risikogruppenidentifizierung unter differentiellen Aspekten unterschiedlicher Behinderungen betrachten. Wir gehen von der Grundannahme aus, dass die 5 Prinzipien von Hobfoll et al. (2007, s.o.) für behinderte Menschen gleichermaßen zu berücksichtigen sind wie bei nichtbehinderten Menschen. Wir gehen davon aus, dass die Tatsache einer Behinderung nicht impliziert, dass die Zugehörigkeit zur Risikogruppe ohne Sichtung von Risiko- und Schutzfaktoren gegeben ist. Wir gehen auch davon aus, dass die Differenzierung in Selbsterholer, Wechsler- und Risikogruppe auch für behinderte Menschen Gültigkeit hat. Allerdings folgern wir, dass Behinderungen Vulnerabilitäten mit sich bringen, die $\mathrm{zu}$ bedenken sind.

In den ersten Handlungsempfehlungen für die PSNV für Menschen mit Behinderungen differenzieren wir zwischen Sinnesbehinderungen, Körperbehinderungen und geistigen Behinderungen. Wir müssen uns konzeptionell darauf einstellen, dass sie kombiniert sein können. Beginnen wir mit den Sinnesbehinderungen.

\subsubsection{Sinnesbehinderungen}

Unter Menschen mit Sinnesbehinderung verstehen wir primär Menschen mit einer Schädigung des Seh- oder Hörvermögens, die nicht durch kompensatorische Hilfsmittel (z.B. Brille oder Hörhilfe) ausgeglichen werden kann. Wahrnehmungs- und Kommunikationseinschränkungen sind somit das zentrale Thema, auf das sich Berufsgruppen der Notfallmedizin einstellen müssen. Menschen mit Hörschädigungen weisen hohe Prävalenzraten hinsichtlich psychischer Störungen auf (Fellinger et al. 2012). Bei sehbehinderten Menschen gibt es keine aussagekräftigen Ergebnisse; es lassen sich lediglich Hinweise auf eine höher ausgeprägte Symptombelastung im Vergleich zu sehenden Menschen finden (Sarimski 2005). Sinnesbehinderte Menschen gelten als besonders vulnerabel (Gerber 2009; Neuhauser et al. 2013; Stout et al. 2004; Sullivan u. Häkkinen 2006). Sinnesbehinderte weisen außerdem ein erhöhtes Risiko auf, sich im Rahmen von Großschadensereignissen nicht in Sicherheit bringen zu können, Gefahren nicht angemessen wahrzunehmen und sich zu verletzen (Legood et al. 2002). Auditive oder visuelle Warnungen und Anweisungen zu Evakuierung oder bestehende Gefahren können nicht wahrgenommen oder umgesetzt werden (Ulmasova et al. 2009).

Hörgeschädigte und Sehbehinderte sind besonders vulnerabel, wenn sie ihre Sinnesbehinderung nicht durch andere Sinnesorgane kompensieren können. 
Bei Hörgeschädigten ist das vor allem in der Dunkelheit, beim Fahren von Fahrzeugen oder anderen Situationen gegeben, in denen das Sichtfeld eingeschränkt ist. Sehbehinderte Menschen sind bei einer lauten Geräuschkulisse besonders empfindsam (Stout et al. 2004; U.S. Department of Education 2008).

Die Notwendigkeit, Handlungsempfehlungen für diese Gruppe zu entwickeln, hat auch die europäische Kommission erkannt. Aus diesem Grunde haben wir das EUProjekt „European Network for Psychosocial Crisis Management - Assisting Disabled in Case of Disaster (EUNAD) " in das Leben gerufen. EUNAD beschäftigt sich mit der speziellen Psychotraumatologie von seh- und hörbehinderten Menschen sowie den speziellen Bedarfen in der PSNV und hat Empfehlungen für die PSNV mit dieser Zielgruppe entwickelt. Ein zentrales Ergebnis von EUNAD ist die Feststellung von grundsätzlich gleichen Bedarfen bei Menschen mit Sinnesbehinderungen und Menschen ohne Sinnesbehinderungen. Es lassen sich lediglich Unterschiede bei der Versorgung und Priorität der Bedarfe feststellen (EUNAD 2014; Gerwinn et al. 2015). Wir gehen gesondert auf die Hör- und Sehbehinderung ein.

\section{Hörbehinderungen}

Für die Akutversorgung (Phase A, s. Abb. 16) sollte von allen Berufsgruppen der Notfallmedizin folgende Empfehlungen im Zusammenhang mit hörgeschädigten Menschen berücksichtigt werden:

1. Menschen mit Hörschädigung gehen unterschiedlich mit ihrem Hörverlust um. Um Schamgefühle und das Gefühl des Bloßstellens zu vermeiden, sollten alle Angebote optional und selbstbestimmt gestaltet sein.

2. Für eine funktionierende Informationsvermittlung sollte das Informationsdefizit durch individuelle Betreuung mithilfe geeigneter Kommunikationsmethoden und unter Berücksichtigung von hörschädigungsspezifischen Kommunikationsanforderungen kompensiert werden.

3. Hörschädigungsspezifische Kommunikationsanforderungen sind:

- mehr Zeit einplanen.

- Kontaktaufnahme sollte visuell (z.B. mit der Hand winken) oder sensitiv (z.B. Stampfen auf dem Boden oder Antippen) erfolgen.

- Körperkontakt hat einen beruhigenden Einfluss und ist in der Gehörlosenkultur verstärkt üblich.

- Aufnehmen und Halten von Augenkontakt signalisiert beiderseitige Aufmerksamkeit. Während der Kommunikation den Blick abzuwenden gilt in der Gehörlosenkultur als unhöflich.

- Bei hochgradig Schwerhörigen ist festzustellen, ob es ein besser hörendes Ohr gibt und sich entsprechend zu positionieren.

v Berücksichtigen, dass hochgradig hörgeschädigte Menschen unterschiedliche kommunikative Fähigkeiten aufweisen. Daher sollte zuerst die individuellen kommunikativen Fähigkeiten erfasst und die jeweilig präferierte Kommunikationsmethode genutzt bzw. bereitgestellt werden.

4. Geeignete Kommunikationsmethoden können sein:

- Lautsprache (einfache und kurze Sätze im lauten und deutlichen Ton formulieren, das Gesicht nicht abwenden, den Mund nicht verdecken),

ㅁ DGS (Gebärdensprachdolmetschen organisieren und über andere Kommunikationsmethoden informieren, dass Gebärdensprachdolmetschen bereitgestellt wird), 
- Nonverbale Kommunikation(z.B. Gesten, Mimik, Körpersprache, Deuten, intuitive Gebärden),

- Schriftsprache (kurze und einfache Sätze nutzen, keine Fremdwörter, Namen/Eigennamen immer aufschreiben).

- Informationsvermittlung durch Sicht auf das Geschehen,

- Bilder (z.B. über standardisierte Karten oder Malen Schmerzen abfragen), Kommunikation über Anwesende (z.B. gebärdensprachkompetente Betroffene) diese Methoden sollte ausschließlich übergangsweise genutzt werden, da kein Eins-zu-Eins Dolmetschen gewährleistet ist (Gerwinn et al. 2015).

Ist ein sicherer Rahmen geschaffen, wie es für die Phase B und C vorauszusetzen ist, so sollte eine funktionierende Informationsvermittlung sichergestellt werden. Der Zugang zu Informationsmaterialien in Schriftsprache, Lautsprache, DGS, Bildern, etc., hörschädigungsspezifische Hilfsmittel wie Batterien für Hörhilfen, Stromversorgung, Schreibmaterial oder eine elektronische Schreibalternative, Mobiltelefon/Smartphone, Laptop/Computer mit Internetverbindung und Kamera, Kärtchen mit Standardfragen und Bildern sowie ein Gebärdensprachalphabet, sollte gewährleistet werden.

Bei dem Einsatz von Stabilisierungsübungen sollten Methoden verwendet werden, die die Informationsaufnahme nicht zusätzlich beeinträchtigen (z.B. keine imaginative Übungen mit geschlossenen Augen oder liegende Übungen). Die Übungen sollten vorgemacht werden. Bislang existiert kein Gebärdensprachdolmetscherbereitschaftsdienst in Deutschland (EUNAD 2014; Gerwinn et al. 2015).

\section{Aus diesem Grund sollten Fachkräfte der PSNV und Leitstellen Kontaktdaten über regional verfügbare Gebärdensprachdolmetscher verfügbar haben.}

\section{Sehbehinderungen}

Für die Akutversorgung (Phase A) sollten folgende Empfehlungen im Zusammenhang mit sehbehinderten Menschen berücksichtigt werden:

1. Menschen mit Sehbehinderung gehen unterschiedlich mit ihrer Behinderung um. Um Schamgefühle und das Gefühl des Bloßstellens zu vermeiden, sollten alle Angebote optional und selbstbestimmt gestaltet sein.

2. Für eine funktionierende Informationsvermittlung:

- Kontaktaufnahme sollte immer auditiv unter Vorstellung von Namen und Profession erfolgen.

- Es sollte immer direkt kommuniziert werden, nicht über die Assistenz.

- Es besteht der Bedarf an detaillierteren mündlichen Informationen (z.B. Beschreibung des äußeren Umfeldes, des aktuellen Geschehens, nächste Schritte) daher muss mehr Zeit eingeplant werden.

- Es sollten keine „emotionalen Ausdrücke“ (z.B. „Oh Gott“, „Neeeeiiiin!!“) genutzt werden, damit das relative Sicherheitserleben nicht zusätzlich geschwächt wird.

3. Wenn blinde Menschen an einen anderen Ort geführt werden müssen:

- Hilfreich ist, Blinde am Ellenbogen fassen zu lassen, einen Schritt vorauszugehen, mündlich auf Hindernisse, Bodenbelagsänderungen, Bürgersteige, Treppen, Türen etc. hinweisen und ein „normales“ Schritttempo zu verwenden. 
Blinde sollten nicht alleine zurückgelassen werden. Wenn dies notwendig wird, sollten Sehbehinderte darüber mündlich informiert und in die Nähe eines festen Gegenstandes positioniert werden. Nach Möglichkeit sollte mitgeteilt werden, wann wieder jemand zurückkommt (Gerwinn et al. 2015).

Ist ein sicherer Rahmen geschaffen, wie es für die Phase B und C vorauszusetzen ist, so sollte eine funktionierende Informationsvermittlung hergestellt werden. Dafür sollten Informationsmaterialien in verschiedenen Kommunikationsmethoden (auditiv, Braille, elektronisch-schriftsprachlich), verfügbar sein. Sehschädigungsspezifische Ressourcen wie Lupe, Blindenstock und Informationen in Braille sollten gewährleistet werden. Wenn eine sensitive Kontaktaufnahme notwendig wird, sollte diese immer vorher angekündigt werden. Ggf. sollte sich rückversichert bzw. eine Cenehmigung eingeholt werden.

Bei dem Einsatz von Stabilisierungsübungen sollten vorzugsweise verbale Methoden genutzt und sehbehinderte Menschen dazu an einen geräuscharmen Ort gebracht werden. Die Erhebung von Risiko- und Schutzfaktoren mit dem KRI muss in einem Interview durchgeführt werden. Offene Fragen sollten gestellt werden, in welcher Form die Sehbehinderung sich auf die Situationsdynamik ausgewirkt hat. Das heißt: Gab es in der akuten Phase Umstände, die das Gefühl von Ohnmacht, Hilf- und Orientierungslosigkeit verstärkt haben?

Für alle Formen der Sinnesbehinderungen gilt, dass die Hilfsverbände so früh wie möglich eingebunden werden sollten.

\subsubsection{Körperbehinderungen}

Unter dieser Gruppe verstehen wir Betroffene, die unter erheblichen Beeinträchtigungen des muskuloskelletalen-Systems leiden. Wir orientieren uns an einer Personengruppe, die an den Rollstuhl gebunden ist. Wir müssen uns insbesondere über die Phase A der Akutversorgung Gedanken machen, da Rollstuhlfahrer auf externe Hilfe bzw. Barrierefreiheit angewiesen sind. In besonderer Form rückt die Notwendigkeit in den Vordergrund, Sicherheit herzustellen. Rollstuhlfahrer haben Barrieren zu überwinden, die für Menschen ohne motorische Beeinträchtigungen problemfrei überwunden werden können. Insbesondere für die Rettung oder Evakuierung von Menschen mit Körperbehinderungen müssen die Ersthelfer mehr Zeit einplanen.

Für die Akutversorgung (Phase A, s. Abb. 16) sollte von allen Berufsgruppen der Notfallmedizin folgende Empfehlungen im Zusammenhang mit motorischen Beeinträchtigungen berücksichtigt werden:

1. Menschen mit Körperbehinderung gehen unterschiedlich mit ihrer Behinderung um. Einige haben große Fähigkeiten entwickelt, mit Hilfsmitteln die Behinderung zu kompensieren. Aus diesem Grunde ist es wichtig, Körperbehinderte auf ihre Beeinträchtigung anzusprechen, um sich ein Bild zu machen, wobei Hilfe benötigt wird.

2. Kommunikationsspezifika sind:

- mehr Zeit einplanen.

- Kontaktaufnahme sollte im Sichtfeld erfolgen, d.h. Sitzhöhe von Rollstuhlfahrern annehmen. 
Körperkontakt kann eine beruhigende aber auch eine erschreckende Wirkung haben. Auf jeden Fall sollte Körperberührung angekündigt sein und nicht außerhalb des Sichtfeldes erfolgen.

Ist ein sicherer Rahmen geschaffen, wie es für die Phase B und C vorauszusetzen ist, so gelten für Menschen mit Körperbehinderungen keine wesentlichen Besonderheiten. In Ersatzunterkünften muss allerdings gesichert sein, dass die Zugänge barrierefrei sind, z.B. müssen Aufzüge funktionstüchtig sein oder Treppenstufen mit Brettern belegt werden. Im Rahmen der Psychosozialen Akuthilfe und mittelfristigen psychosozialen Hilfen muss aber der möglichen Immobilität und eventuellen Bedarfen besondere Aufmerksamkeit geschenkt werden. Gegenstand der Psychoinformation muss dementsprechend auch sein, welche besonderen Hilfestellungen für Menschen mit motorischen Behinderungen notwendig und welche gegeben sind. Bei der Vermittlung von Stabilisierungsübungen ist darauf zu achten, dass ggf. alle Übungen im Sitzen durchführbar sein müssen. Über die reguläre Anwendung des KRI hinaus ist zu erfragen, ob die Tatsache der Behinderungen situative Risikofaktoren verstärkt und ob z.B. die Mobilisation von sozialen Netzwerken gesichert ist.

\subsubsection{Geistige Behinderungen}

Die geistigen Behinderungen können von einer leichten bis zu einer schweren Intelligenzminderungen gegeben sein. Da eine häufige Ursache die Sauerstoffunterversorgung unter der Geburt ist (Asphyxie), sind die geistigen Behinderungen häufig mit Spastiken kombiniert. In diesen und vergleichbaren Fällen sprechen wir über komplexe Behinderungen.

Der Umgang mit geistig behinderten Menschen, in komplexen Gefahren- und Schadenslagen und in der PSNV, stellt je nach Grad der Einschränkung eine besondere Herausforderung dar und es gibt bis dato kaum spezifische Konzepte. Zu beantworten ist zunächst die Frage, was wir in der Akutphase (Phase A) tun können?

$\mathrm{Zu}$ berücksichtigen ist zum einen, dass die Impulskontrolle bei Menschen mit geistigen Behinderungen in der Regel gemindert ist. Es kann zu Erregungsstürmen kommen, die dazu führen, dass die Situation nicht kontrollierbar ist und die medizinische Versorgung gefährdet sein kann. Tatsächlich sind Notärzte u.U. vor die Entscheidung gestellt, durch Akutmedikationen Kontrollierbarkeit herzustellen.

Wir müssen bedenken, dass die Beruhigung am ehesten durch Betreuungspersonen aus dem persönlichen Umfeld möglich ist, die Kenntnis von individuellen Gewohnheiten der Menschen mit geistigen Behinderungen haben, die der Schlüssel zur Beruhigung sein können. Möglicherweise stehen vertraute Betreuungspersonen in der Akutsituation aber nicht so schnell zur Verfügung und Einsatzkräfte und Psychosoziale Akuthelfer sehen sich mit der Aufgabe konfrontiert, die Menschen zu beruhigen und zu versorgen.

Aus Erfahrungsberichten zur psychosozialen Versorgung von Menschen mit geistiger Behinderung in komplexen Gefahren- und Schadenslagen lässt sich ableiten, dass basale Maßnahmen der psychischen Ersten Hilfe analog zum Umgang mit Kindern am ehesten zum Erfolg führen. Hierzu gehören z.B.

- sich mit Vornamen vorstellen

- Kopfbedeckung abnehmen 
- auf die Ebene der Person gehen

n eine Person abstellen, die für die Betroffene zuständig ist und möglichst bei ihr bleiben und sie durchgängig betreuen kann

- beruhigend und langsam sprechen, klare und einfache Sätze formulieren

- der betroffenen Person in einfachen Worten mitteilen, was passiert und was passieren wird

- Mut zusprechen und einen positiven Ausgang ankündigen

- die Person loben und deren Bewältigungsstrategien betonen

- Gefühle nicht versuchen auszureden, z.B. Angst

- die Aufmerksamkeit umlenken, z.B. auf Bilder, Gegenstände

- der Einsatz von „Plüschtierkollegen“ als Aufpasser, Erklärer

- taktile Erfahrungen ermöglichen, z.B. vertrauten Gegenstand mitnehmen

- Körperkontakt einsetzen als haltgebende, sichernde Erfahrung

- Primärbedürfnisse befriedigen durch Getränke und Nahrungsmittel

Auch für die weitere Vorgehensweise der mittel- und langfristigen psychosozialen Versorgung gibt es kaum Verfahrensvorschläge. Inwieweit vereinfachte psychoinformative Elemente und Anleitungen zur Selbsthilfe aufgenommen werden können, ist maßgeblich abhängig vom Grad der Behinderung und von der Fähigkeit zu verbalisieren und verbale Informationen zu verarbeiten. Dies gilt auch für die Umsetzung einfacher Stabilisierungsübungen. Hier wird es zukünftig notwendig sein, den psychosozialen „Werkzeugkoffer“ um einfache Übungselemente zu erweitern, die weniger auf kognitive Komplexität oder Imagination abzielen, sondern konkret mit Hilfsmitteln auf der Handlungsebene ansetzen.

Die Erfassung von Risiko- und Schutzfaktoren ist zu komplex und unser Wissen über die Verlaufstypen aktuell noch zu gering, als dass wir gezielte Empfehlungen aussprechen könnten. Nichtsdestotrotz lässt sich ein Teil der im KRI erhobenen Risikound Schutzfaktoren durchaus direkt oder fremdanamnestisch erheben. Die konkrete Erfassung eines Risiskoprofiles bedarf jedoch zukünftiger Forschung.

Es ist bei Notfallbeteiligten mit geistiger Behinderung wichtig, vertraute Bezugspersonen so schnell wie möglich zu mobilisieren, die aus ihrer Betreuungskenntnis und Erfahrung Orientierung vermitteln können.

\subsection{Ausblick}

Wir kommen zu unserem Ausblick und konstatieren, dass die Verabschiedung der UN-BRK auch unsere Konzepte der PSNV auf die Probe stellt, ob wir die besondere Ausgangssituation von Menschen mit Behinderungen berücksichtigt haben. Wir haben das TGIP um Handlungsempfehlungen ergänzt, die hilfreich seien können, Schadenslagen zu bewältigen. Sicherlich ist für Menschen mit einer Sinnesbehinderung, motorischen Beeinträchtigung und einer geistigen Behinderung in der Akutphase die Gefahr groß, dass eine Potenzierung von ereignisbezogenen Risikofaktoren eintritt. Wir ziehen folgende Schlussfolgerungen:

1. Für alle Gruppen der Behinderungen sind die 5 Prinzipien der Frühintervention von Hobfoll et al. zu berücksichtigen. 
2. Nach bisherigem Kenntnisstand gehen wir davon aus, dass generelle Risikound Schutzfaktoren auch für Menschen mit Behinderungen Gültigkeit haben.

3. Unsere Handlungsempfehlungen berücksichtigen spezifische Vulnerabilitäten, die mit der Behinderung verbunden sind.

4. Oft verfügen Menschen mit Behinderungen über gute Anbindungen an Betroffenenorganisationen, die als soziales Netzwerk mit besonderer Fachkunde von Berufsgruppen der Notfallmedizin mobilisiert werden sollten.

5. Wir konstatieren, dass es keine empirischen Daten über spezielle Risikofaktoren von Sinnesbehinderungen, Körperbehinderungen oder geistigen Behinderungen gibt.

Die wirksame Umsetzung von Handlungsempfehlungen setzt voraus, dass alle Berufsgruppen der Notfallmedizin für die Belange der Sinnes-, körperlichen und geistigen Behinderungen sensibilisiert werden. Diese Sensibilisierung sollte durch die Einbindung von Handlungsempfehlungen in die Curricula der Notfallmedizin durch Selbsterfahrung, Wissensvermittlung (z.B. kommunikative Fähigkeiten, Gehörlosenkultur, Blindenhilfsmittel) und Simulationstrainings erfolgen. Hierbei haben wir die Erfahrung gemacht, dass die Behindertenverbände durchaus über Experten verfügen (EUNAD 2014; Gerwinn et al. 2015), die Hilfestellung leisten können.

\section{Abkürzungen}

Multidisciplinary Guidelines

Posttraumatische Belastungsstörung

Target Group Intervention Program

UN-Behindertenrechtskonvention

Kölner Risikoindex
(MG)

(PTBS)

(TGIP)

(UN-BRK)

(KRI)

\section{Literatur}

Bachmann W (2013) Katastrophenschutz für hörbehinderte Menschen. Eine Dokumentation von Wolfgang Bachmann. 2. Aufl. Berlin, Deutscher Gehörlosen Bund e.V. http://www.barrierefreiheit.de/tl_files/bkb-downloads/Projekte/katastrophenschutz/bkb_dgb_katastrophenschutz_130924.pdf (Zugriff am 28.08.2014

Bering R (2011) Verlauf der Posttraumatischen Belastungsstörung. Grundlagenforschung, Prävention, Behandlung. 2. erweiterte Auflage. Shaker Verlag, Aachen.

Bering R, Fischer G (2005) Kölner Risiko Index (KRI). In: Strauß B, Schuhmacher I (Hrsg.) Klinische Interviews und Ratingskalen. Göttingen, Hogrefe, S. 216-221

Bering R, Schedlich C, Zurek G (2015a) Großschadenslagen als potentiell traumatisierende Ereignisse. In: Seidler GH, Freyberger H), Maercker A (Hrsg.) Handbuch der Psychotraumatologie. 2. Auflage. Stuttgart, Klett-Cotta, S. 555-569

Bering R, Schedlich C, Zurek G (2015b) Situationstypologien der Psychosozialen Notfallversorgung. In: Seidler GH, Freyberger HJ, Maercker A (Hrsg.) Handbuch der Psychotraumatologie. 2. Auflage. Stuttgart, Klett-Cotta, S. 749-763

Bering R, Schedlich C, Zurek G, Grittner G, Kamp M, Fischer G (2008) Manual for implementing the Cologne Risk Index-Disaster in the context of major loss situations. http://www.eutopa-info.eu/index.php?id=69, Accessed: (Zugriff am 12.3.2011)

Bundesamt für Bevölkerungsschutz und Katastrophenhilfe (2011a) BBK-Glossar, Ausgewählte zentrale Begriffe des Bevölkerungsschutzes. Bonn. http://www.bbk.bund.de/SharedDocs/Downloads/BBK/DE/Publikationen/Praxis_Bevoelkerungsschutz/Band_8_Praxis_BS_BBK_Glossar.pdf?_blob=publicationFile (Zugriff am 21.06.2014) 
Bundesamt für Bevölkerungsschutz und Katastrophenhilfe (2011b) Psychosoziale Notfallversorgung: Qualitätsstandards und Leitlinien, Teil I und II. Bonn. http://www.bbk.bund.de/SharedDocs/Downloads/BBK/DE/ Publikationen/Praxis_Bevoelkerungsschutz/Band_7_Praxis_BS_PSNV_Qualit_stand_Leitlinien_Teil_1_2. pdf?_blob=publicationfile (Zugriff am 21.06.2014)

EUNAD (Hrsg.) (2014) EUNAD. European Network for Psychosocial Crisis Management - Assisting Disabled in Case of Disaster. http://eunad-info.eu/home.html (Zugriff am 06.12.2014)

Fellinger J, Holzinger D, Pollard R (2012) Mental health of deaf people. The Lancet 379: 1037-1044

Fischer G (2003) Neue Wege aus dem Trauma. Erste Hilfe bei schweren seelischen Belastungen. Düsseldorf: Walther

Fischer G, Riedesser P (2009) Lehrbuch der Psychotraumatologie. 4. Aufl. München, Reinhardt

Gerber E (2009) Describing Tragedy: The Information Access Needs of Blind People in Emergeny-related Circumstances. Human Organization 68: 73

Gerwinn F, Kaul T, Bering R, Schedlich C (2015) Psychosoziale Notfallversorgung nach Großschadensereignissen von Menschen mit hochgradiger Hörschädigung. In Vorbereitung

Hobfoll SE, Watson P, Bell CC, Bryant RA, Brymer M), Friedman M), Friedman M, Gersons BP, de Jong JT, Layne CM, Maguen S, Neria Y, Norwood AE, Pynoos RS, Reissman D, Ruzek II, Shalev AY, Solomon Z, Steinberg AM, Ursano RJ (2007) Five essential elements of immediate and mid-term mass trauma intervention. Empirical evidence. Psychiatry 70(4): 283-315; discussion 316-69

IMPACT (2007) European Multidisciplinary Guideline. Early psychosocial interventions after disaster, terrorism and other shocking events. Impact, Dutch knowledge \& advice centre of post-disaster psychosocial care

Legood R, Scuffham P, Cryer C (2002) Are we blind to injuries in the visually impaired? A review of the literature. Inj Prev 8: 155-160

NATO \& OTAN (2008) Psychosocial care for people affected by disasters and major incidents. Non-binding guidance. NATO Joint Medical Committee

Neuhauser L, Ivey S, Huang D, Engelman A, Tseng W, Dahrouge D, Gurung S, Kealey M (2013) Availability and Readability of Emergency Preparedness Materials for Deaf and Hard-of-Hearing and Older Adult Populations: Issues and Assessments. PLoS ONE 8(2): e55614

Reddemann L (2001) Imagination als heilsame Kraft. Stuttgart: Pfeiffer bei Klett Cotta

Sarimski K (2005) Psychische Störungen bei behinderten Kindern und Jugendlichen. Göttingen, Hogrefe

Schedlich C, Zurek G, Bering R (2008) Manual for target group intervention within the scope of major losses and disasters. http://www.eutopa-info.eu/index.php?id=69 (Zugriff am 12.03.2011)

Stout C, Heppner C, Brick K (2004) Emergency Preparedness and Emergency Communication Access. Lessons Learned Since 9/11 and Recommendations. https://tap.gallaudet.edu/Emergency/Nov05Conference/EmergencyReports/DHHCANEmergencyReport.pdf (Zugriff am 24.12.2014)

Sullivan MT, Häkkinen HT (2006) Disaster preparedness for vulnerable populations: Determining effective strategies for communicating risk, warning, and response. In Third Annual Magrann Research Conference at Rutgers University (Vol. 4, pp. 1-36). Research Inst. of NRCPD. http://magrann-conference.rutgers.edu/2006/_ papers/sullivan.pdf (Zugriff am 11.10.2014)

U.S. Department of Education (Hrsg.) (2008) Emergency Management Research and People With Disabilities: A Resource Guide, Washington D.C.

Übereinkommen der Vereinten Nationen über die Rechte von Menschen mit Behinderungen, UN-BRK (2009) http://www.institut-fuer-menschenrechte.de/fileadmin/user_upload/

Ulmasova I, Silcock N, Schranz B (2009) Mainstreaming Disability into Disaster Risk Reduction: A Training Manual. Nepal: Handicap International Nepal, S. 1-87

Zurek G, Schedlich C, Bering R (2008) Manual for trauma-based psychoeducation for victims of disasters. Available: http://www.eutopa-info.eu/index.php?id=69, (Zugriff am 12.03.2011) 


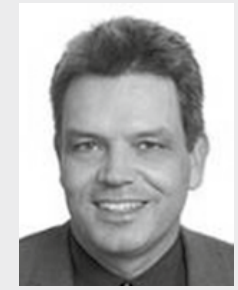

\section{Prof. Dr. med. Dipl..Psych. Robert Bering}

Professor Robert Bering studierte an der Universität zu Köln Humanmedizin und Psychologie. Er verbrachte zwei Forschungssemester am Institut für Molekulare Neuropathologie an der Universität Kopenhagen und promovierte 1995. Am Kreiskrankenhaus Gummersbach absolvierte er seine klinische Ausbildung zum Facharzt für Psychiatrie und Psychotherapie. Von 2000 bis 2008 war er wissenschaftlicher Mitarbeiter am Institut für Klinische Psychologie und Psychologische Diagnostik der Universität zu Köln und habilitierte sich. Aktuell ist Herr Prof. Bering Chefarzt des Nordrheinischen Netzwerks für Psychotraumatologie der Alexianer Krefeld GmbH. In Verbindung mit der Ausgestaltung eines Masterstudiengangs für Rehabilitationswissenschaften an der Universität zu Köln wurde er 2011 zum außerplanmäßigen Professor ernannt.

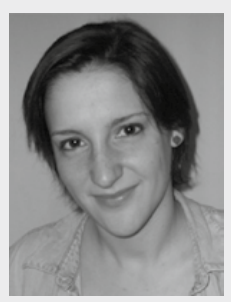

\section{Florentine Gerwinn, M.A. Reha.-wis}

Frau Florentine Gerwinn studierte Rehabilitationswissenschaften (M.A.). Am Zentrum für Psychotraumatologie Köln-Süd, Alexianer Krefeld GmbH, ist sie im Sozialdienst und Rehabilitations- und Teilhabemanagement tätig. Mitarbeit in EUNAD. Schwerpunkt: Psychotraumatologie.

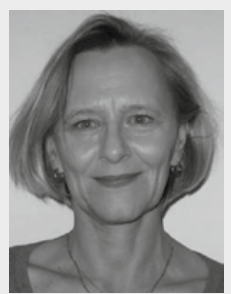

\section{Claudia Schedlich, Dipl..Psych.}

Frau Claudia Schedlich ist im Bundesamt für Bevölkerungsschutz und Katastrophenhilfe in Bonn, Referat „Psychosoziales Krisenmanagement“ als Psychologin, Psychotherapeutin mit dem Schwerpunkt Psychotraumatherapie tätig. Forschungsgebiet und Lehre: Psychotraumatologie. Kooperationspartner im Projekt EUNAD.

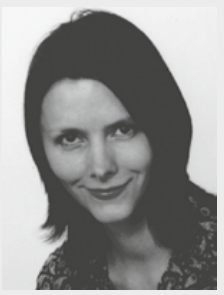

\section{Gisela Zurek, Dipl.-Psych.}

Frau Gisela Zurek arbeitet als wissenschaftliche Mitarbeiterin im Projekt EUNAD am Zentrum für Psychotraumatologie, Alexianer Krefeld GmbH. Forschungsgebiet und Lehre: Psychotraumatologie, Trauma-Nachsorge im Bereich öffentliche Gesundheitsfürsorge. 